\title{
Semisimple Graded Rings
}

\section{N. Balaba, E. N. Krasnova}

Leo Tolstoy Tula State Pedagogical University, Russia, 300026, Tula, Lenina pr., 125, ibalaba@mail.ru, KrasnovaEN.ne@yandex.ru The graded version of Wedderburn-Artin theorem is obtained. It gives description of semisimple $G$-graded ring for arbitrary group $G$. Homological classification of graded semisimple rings is given.

Key words: graded rings, graded modules, semisimple rings.

\section{References}

1. Nǎstǎsescu C., Oystaeyen F. van. Method of graded rings. Berlin, Springer, 2004, $295 \mathrm{p}$.

2. Hwang Y.-S., Wadsworth A. R. Correspondences between valued division algebras and graded division algebras. J. Algebra, 1998, vol. 220, pp. 73-114.

3. Bahturin Yu. A. Zaicev M. V., Sehgal S. K. Finite-dimensional simple graded algebras. Sbornik : Mathematics, 2008, vol. 199, no. 7, pp. 965-983. DOI:10.1070/SM2008v199n07ABEH003949

4. Balaba I. N. Mikhalev A. V. Isomorphisms of graded endomorphism rings of graded modules close to free ones. J. Math. Sci., 2009, vol. 156, no. 2, pp. 209-218.

5. Balaba I. N. Graduirovannye prostye artinovy kol'tsa [Graded simple artinian rings]. Algebra $i$ matematicheskaia logika : materialy mezhdunar. konf., posviashch. 100-letiiu so dnia rozhdeniia prof. V. V. Morozova [Algebra and Mathematical Logika : Trans. Intern. Confer., dedicated to 100th anniversary of V. V. Morozov]. Kazan, 2011, pp. 43-44 (in Russian).

6. Liu S.-X., Beattie M., Fang H. Graded division rings

and the Jacobson density theorem. J. Beijing Normal University (Natural Science), 1991, vol. 27, no. 2, pp. 129-134.

7. Balaba I. N. Izomorfizmy graduirovannykh kolets lineinykh preobrazovanii graduirovannykh vektornykh prostranstv [Isomorphisms of graded rings of linear transformations of graded vector spaces]. Chebyshevckiy sbornik, 2005, vol. 6. no. 4(16), pp. 6-23 (in Russian).

8. Dǎscǎlescu S., Ion B., Nǎstǎsescu C., Rios Montes J. Group gradings on full matrix rings. J. Algebra, 1999, vol. 220 , pp. 709-728.

9. Bahturin Yu. A., Sehgal S.K., Zaicev M.V. Group graging on associative algebras. J. Algebra, 2001, vol. 241, pp. 677-698.

10. Bahturin Ju. A., Zaicev M. V. Group gradings on matrix algebras. Canad. Math. Bulletin, 2002, vol. 45, pp. 499-508.

11. Zalesskii A. E., Mikhalev A. V. Group rings. J. of Soviet Math., 1975, vol. 4, no. 1, pp. 1-78.

УДК 501.1

\section{О МНОГООБРАЗИЯХ ГРУППОИДОВ ОТНОШЕНИЙ С ДИОФАНТОВЫМИ ОПЕРАЦИЯМИ}

\author{
Д. А. Бредихин \\ Доктор фризико-математических наук, профрессор кафредры математики и моделирования, Саратовский государственный \\ технический университет им. Гагарина Ю. А., bredikhin@mail.ru \\ В работе находятся базисы тождеств многообразий, порожденных классами группоидов бинарных отношений с диосранто- \\ выми операциями.
}

Ключевые слова: алгебры отношений, диосрантовые операции, тождества, многообразия, группоиды.

\section{ВВЕДЕНИЕ}

Множество бинарных отношений $\Phi$, замкнутое относительно некоторой совокупности $\Omega$ операций над ними, образует алгебру $(\Phi, \Omega)$, называемую алгеброй отношений. Теория алгебр отношений является существенной составной частью современной общей алгебры и алгебраической логики. Основы абстрактно-алгебраического подхода к изучению алгебр отношений были заложены в работах 
A. Тарского (A. Tarski) [1,2]. Как правило, операции над отношениями задаются с помощью формул логики предикатов первого порядка. Такие операции называются логическими. Логические операции могут быть классифицированы по виду задающих их формул. Операция называется диофантовой [3] (в другой терминологии примитивно-позитивной [4]), если она может быть задана с помощью формулы, которая в своей предваренной нормальной форме содержит лишь операции конъюнкции и кванторы существования. Диофантовы операции допускают описания с помощью графов [3-5]. Эквациональные и квазиэквациональные теории алгебр отношений с диофантовыми операциями описаны в работах [5-7].

Предметом нашего рассмотрения будут алгебры отношений с одной бинарной диофантовой операцией, то есть группоиды бинарных отношений. Классификацию бинарных диофантовых операций над отношениями можно найти в [8]. Рассмотрение бинарных операций над отношениями играет в алгебраической логике предикатов роль, аналогичную роли бинарных булевых функций в пропозициональной логике высказываний. Поэтому естественен интерес к алгебраическим свойствам указанных операций, в частности, к свойствам, выразимым тождествами. Это приводит к необходимости изучения многообразий, порожденных различными классами группоидов бинарных отношений. Некоторые результаты в этом направлении можно найти в работах [8-10].

\section{1. ФОРМУЛИРОВКА РЕЗУЛЬТАТОВ}

Группоидом называется алгебра $(A, \cdot)$ с одной бинарной операцией.

Сосредоточим свое внимание на следующей диофантовой операции над бинарными отношениями, определяемой формулой

$$
\rho * \sigma=\{(x, y) \in X \times X:(\exists z, w)(x, z) \in \rho \wedge(z, w) \in \sigma\},
$$

где $\rho$ и $\sigma-$ бинарные отношения, заданные на множестве $X$. Заметим, что отношение $\rho * \sigma$ представляет собой результат применения операции цилиндрофикации [11] к произведению $\rho \circ \sigma$ бинарных отношений $\rho$ и $\sigma$.

Алгебры отношений вида $(\Phi, *)$ является группоидом бинарных отношений.

Для заданного множества $\Omega$ операций над бинарными отношениями обозначим через $R\{\Omega\}$ класс алгебр, изоморфных алгебрам отношений с операциями из $\Omega$. Пусть $\operatorname{Var}\{\Omega\}-$ многообразие, порожденное классом $R\{\Omega\}$.

Теорема. Группоид $(A, \cdot)$ принадлежит многообразию $\operatorname{Var}\{*\}$ тогда и только тогда, когда он удовлетворяет тождествам:

$$
\begin{gathered}
(x y) x=x y, \\
(x y) y=x y, \\
\left(x^{2} y\right) z=\left(x^{2} z\right) y, \\
\left(x y^{2}\right) z=x\left(y^{2} z\right)
\end{gathered}
$$

и для каждого натурального $k>2$ тождеству:

$$
\left(x _ { i _ { 1 } } \left(x _ { i _ { 2 } } \left(\ldots ( x _ { i _ { k - 2 } } ( x _ { i _ { k - 1 } } x _ { i _ { k } } ) \ldots ) \left(x _ { i _ { 1 } } \left(x _ { i _ { 2 } } \left(\ldots\left(x_{i_{k-3}}\left(x_{i_{k-2}} x_{i_{k-1}}\right) \ldots\right)=x_{i_{1}}\left(x_{i_{2}}\left(\ldots\left(x_{i_{k-1}} x_{i_{k}}\right) \ldots\right) .\right.\right.\right.\right.\right.\right.\right.
$$

Заметим, что приведенный в теореме базис тождеств является бесконечным. В связи с этим естественно возникает следующая проблема, в настоящее время остающаяся открытой.

Проблема. Является ли многообразие $\operatorname{Var}\{*\}$ конечно базируемылм?

\section{2. ДОКАЗАТЕЛЬСТВО}

Доказательство теорем основывается на результатах работы [5]. Разобьем его на ряд последовательных шагов. 
Шаг 1. Приведем ряд определений и обозначений, используемых в дальнейшем изложении, и сформулируем необходимый результат из работ [5].

Пусть $\operatorname{Rel}(U)$ - множество всех бинарных отношений на $U$. Всякая формула $\phi\left(z_{0}, z_{1}, r_{1}, \ldots, r_{m}\right)$ логики предикатов первого порядка с равенством, содержащая $m$ бинарных предикатных символов $r_{1}, \ldots, r_{m}$ и две свободные индивидуальные переменные $z_{0}, z_{1}$, определяет $m$-арную операцию $F_{\varphi}$ на $\operatorname{Rel}(U)$ :

$$
F_{\varphi}\left(R_{1}, \ldots, R_{m}\right)=\left\{(x, y) \in U \times U: \varphi\left(x, y, R_{1}, \ldots, R_{m}\right)\right\}
$$

где $\varphi\left(x, y, R_{1}, \ldots, R_{m}\right)$ означает, что формула $\varphi$ выполняется, если $z_{0}, z_{1}$ интерпретируются как $x, y$ и $r_{1}, \ldots, r_{m}$ интерпретируются как отношения $R_{1}, \ldots, R_{m}$ из $\operatorname{Rel}(U)$.

Операция над бинарными отношениями называется диофантовой [3] (в другой терминологии примитивно-позитивной [4]), если она может быть определена формулой, содержащей в своей записи лишь кванторы существования и операцию конъюнкции. Диофантовы операции могут быть описаны с помощью графов [3-5].

Обозначим через $N$ множество всех натуральных чисел. Помеченным графом назовем пару $(V, E)$, где $V$ - конечное множество, называемое множеством вершин, и $E \subset V \times N \times V-$ тернарное отношение. Тройку $(u, k, v) \in E$ будем называть ребром графа, идущим из вершины $u$ в вершину $v$, помеченным меткой $k$, и графически изображать следующим образом: $u \cdot \stackrel{k}{\rightarrow} \cdot v$. Мы также будем говорить, что вершины $u$ и $v$ инцидентны ребру $(u, k, v)$.

Под двухполюсником мы понимаем помеченный граф с парой выделенных вершин, то есть систему вида $G=(V, E$, in, out $)$, где $(V, E)$ - помеченный граф; in $=i n(G)$ и out $=$ out $(G)$ - две выделенные вершины (не обязательно различные), называемые входом и выходом двухполюсника соответственно.

Понятие изоморфизма помеченных графов и двухполюсников определяется естественным образом. В дальнейшем все графы будут рассматриваться с точностью до изоморфизма. Мы также будем отождествлять двухполюсники, различающиеся лишь числом изолированных вершин, отличных от его входа и выхода.

Пусть $F=F_{\varphi}-$ диофантова операция, задаваемая формулой $\varphi$. С этой операцией может быть ассоциирован двухполюсник $G=G(F)=G(\varphi)$, определяемый следующим образом: $V(G)-$ множество всех индексов индивидуальных переменных, входящих в формулу $\varphi ; \operatorname{in}(G)=0$, out $(G)=1$; $(i, k, j) \in E(G)$ тогда и только тогда, когда атомарная формула $r_{k}\left(z_{i}, z_{j}\right)$ входит в $\varphi$; если формула $z_{i}=z_{j}$ входит в $\varphi$, то вершины $i$ и $j$ отождествляются.

Заметим, что двухполюсник, соответствующий операции $*$, задается следующим образом:

$$
\text { in }=\cdot \stackrel{1}{\rightarrow} \cdot \stackrel{2}{\rightarrow} \cdot \quad \cdot=\text { out } .
$$

Пусть $G=(V, E$, in, out $)$ и $G_{k}=\left(V_{k}, E_{k}, i n_{k}\right.$, out $\left._{k}\right)(k=1, \ldots, m)$ - двухполюсники с попарно непересекающимися множествами вершин. Назовем композицией этих двухполюсников новый двухполюсник $G\left(G_{1}, \ldots, G_{m}\right)$, определяемый следующим образом [4]: возьмем двухполюсник $G$ и заменим каждое его ребро $(u, k, v) \in E$ на двухполюсник $G_{k}$, отождествляя при этом вершину $i n_{k}$ с вершиной $u$ и вершину out $_{k}$ с вершиной $v$.

Рассмотрим множество $\Omega=\left\{F_{\varphi_{1}}, \ldots, F_{\varphi_{n}}\right\}$ диофантовых операций над отношениями, и пусть $A=\left(A, f_{1}, \ldots, f_{n}\right)$ - универсальная алгебра соответствующего типа. Положим $G_{1}=G\left(\varphi_{1}\right), \ldots$, $G_{n}=G\left(\varphi_{n}\right)$.

Для всякого терма $p$ алгебры $A$ определим следующим индуктивным образом двухполюсник $G(p)=(V(p), E(p), \operatorname{in}(p)$, out $(p))$ :

1) если $p=x_{k}$, то $G(p)$ представляет собой двухполюсник вида $\mathrm{in} \cdot \stackrel{k}{\rightarrow} \cdot$ out;

2 ) если $p=f_{k}\left(p_{1}, \ldots, p_{m}\right)$, то $G(p)$ есть композиция $G_{k}\left(G\left(p_{1}\right), \ldots, G\left(p_{m}\right)\right)$.

Обозначим через $\operatorname{pr}(\mathrm{E})$ множество всех вершин помеченного графа, которые инцидентны хотя бы одному ребру. Пусть даны два помеченных графа $\left(V_{1}, E_{1}\right)$ и $\left(V_{2}, E_{2}\right)$. Отображение $f: \operatorname{pr}\left(E_{2}\right) \rightarrow \operatorname{pr}\left(E_{1}\right)$ называется гомоморфизмом $E_{2}$ в $E_{1}$, если $(f(u), k, f(v)) \in E_{1}$ для всякой тройки $(u, k, v) \in E_{2}$. 
Пусть $G_{1}=\left(V_{1}, E_{1}, i n_{1}\right.$, out $\left._{1}\right)$ и $G_{2}=\left(V_{2}, E_{2}, i n_{2}\right.$, out $\left._{2}\right)$ - двухполюсники. Отображение $f: V_{2} \rightarrow V_{1}$ называется гомоморфизмом из $G_{2}$ в $G_{1}$, если $f\left(i n_{2}\right)=i n_{1}, f\left(\right.$ out $\left._{2}\right)=$ out $_{1}$ и $(f(u), k, f(v)) \in E_{1}$ для всякой тройки $(u, k, v) \in E_{2}$.

Мы будем писать $E_{1} \prec E_{2}\left(G_{1} \prec G_{2}\right)$, если существует гомоморфизм из $E_{2}$ в $E_{1}$ (из $G_{2}$ в $G_{1}$ ), и $E_{1} \cong E_{2}\left(G_{1} \cong G_{2}\right)$, если $E_{1} \prec E_{2}$ и $E_{2} \prec E_{1}\left(G_{1} \prec G_{2}\right.$ и $\left.G_{2} \prec G_{1}\right)$.

Обозначим через $E q\{\Omega\}$ эквациональную теорию класса $R\{\Omega\}$. Теперь мы готовы сформулировать основной результат работы [5]: тождество $p=q$ принадлежит эквациональной теории $E q\{\Omega\}$ тогда и только тогда, когда $G(p) \cong G(q)$.

Шаг 2. Рассмотрим счетное множество индивидуальных переменных $X=\left\{x_{1}, \ldots, x_{n}, \ldots\right\}$. Напомним, что термы группоида определяются следующим индуктивным образом: всякая индивидуальная переменная является термом; если $p_{1}$ и $p_{2}$ - термы, то выражение $\left(p_{1} p_{2}\right)$ является термом, называемым произведением термов $p_{1}$ и $p_{2}$. В дальнейшем внешние скобки в записи термов, как правило, будут опускаться. Множество $\Xi$ всех термов относительно операции произведения термов образует счетно порожденную свободную алгебру в классе всех группоидов.

Обозначим через $\Sigma$ эквациональную теорию класса группоидов, удовлетворяющих тождествам $(1)-(4)$ и $\left(5_{k}\right)$. Для термов $p_{1}$ и $p_{2}$ из $\Xi$ будем писать $p_{1} \cong p_{2}$, когда тождество $p_{1}=p_{2}$ принадлежит $\Sigma$. Отношение $\cong$ является отношением конгруэнции группоида $\Xi$, а фактор группоид $\Xi / \cong$ является свободным счетно порожденным группоидом в многообразии, задаваемым тождествами $(1)-(4)$ и $\left(5_{k}\right)$.

Пусть $(A, \cdot)$ - группоид, удовлетворяющий тождествам (1)-(4). Тогда он удовлетворяет тождествам:

$$
\begin{aligned}
(x y) & =(x y)^{2}, \\
((x y) z) t & =((x y) t) z, \\
(x(y z)) t & =x((y z) t) .
\end{aligned}
$$

Действительно, используя тождество (1), получаем $(x y)=(x y) x=((x y) x)(x y)=(x y)(x y)=(x y)^{2}$. Используя тождества (3), (6), получаем $((x y) z) t=\left((x y)^{2} z\right) t=\left((x y)^{2} t\right) z=((x y) t) z$. Используя тождества (4), (6), получаем $(x(y z)) t=\left(x(y z)^{2}\right) t=x\left((y z)^{2} t\right)=x((y z) t)$.

Замечание 1. Из тождества (8) следует, что подгруппоид $\left(A^{2}, \cdot\right)$, где $A^{2}=\{a b: a, b \in A\}-$ множество разложимых элементов, является полугруппой и, следовательно, скобки, указывающие порядок выполнения действий в произведении элементов из $A^{2}$, могут быть расставлены произвольным образом или просто опущены. В дальнейшем мы будем пользоваться этим свойством без особых упоминаний.

Обозначим через $\Lambda_{k}$, где $k \geq 1$, множество термов вида $x_{i_{1}}\left(x_{i_{2}}\left(\ldots\left(x_{i_{k-1}} x_{i_{k}}\right) \ldots\right)\right.$. Положим

$$
\tilde{\Lambda}_{k}=\bigcup\left\{\Lambda_{i}: i \geq k\right\}
$$

Лемма 1. Для любого терма $p \in \Xi$ существуют такие термь $p_{1}, p_{2}, \ldots, p_{m}$ из $\tilde{\Lambda}_{1}(m \geq 1)$, ито $\left.\left.p \cong\left(\ldots\left(p_{1} p_{2}\right) p_{3}\right) \ldots\right) p_{m-1}\right) p_{m}, u p_{1} \in \tilde{\Lambda}_{2}$, если $p \notin \Lambda_{1}$.

Доказательство. Покажем сначала, что если $\left.\left.p \cong\left(\ldots\left(p_{1} p_{2}\right) p_{3}\right) \ldots\right) p_{m-1}\right) p_{m}$ и $p \notin \Lambda_{1}$, то без ограничения общности можно считать, что $p_{1} \in \tilde{\Lambda}_{2}$. Действительно, если $p \notin \Lambda_{1}$ и $p_{1} \in \Lambda_{1}$, то $m>1$ и $p_{1} p_{2} \in \tilde{\Lambda}_{2}$. Тогда, пологая $\tilde{p}_{1}=p_{1} p_{2}$, получим требуемое представление $\left.\left.\left(\ldots\left(\tilde{p}_{1} p_{3}\right) p_{4}\right) \ldots\right) p_{n-1}\right) p_{m}$ терма $p$.

Утверждение очевидно для $p \in \Lambda_{1}$. Пусть теперь $\left.\left.p=\left(\ldots\left(p_{1} p_{2}\right) p_{3}\right) \ldots\right) p_{m-1}\right) p_{m}$ и $q=\left(\ldots\left(q_{1} q_{2}\right)\right.$ $\left.\left.\left.q_{3}\right) \ldots\right) q_{n-1}\right) q_{n}$.

Если $n=1$, то $\left.\left.\left.p q=\left(\ldots\left(p_{1} p_{2}\right) p_{3}\right) \ldots\right) p_{m-1}\right) p_{m}\right) q_{1}$, то есть произведение $p q$ имеет требуемое представление. Если $n>1$, то, учитывая, что $q_{1} \in \tilde{\Lambda}_{2}$, и, используя тождества (8), получаем:

$$
\left.\left.\left.\left.\left.\left.p q=\left(\ldots\left(p_{1} p_{2}\right) p_{3}\right) \ldots\right) p_{m-1}\right) p_{m}\right)\left(\ldots\left(q_{1} q_{2}\right) q_{3}\right) \ldots\right) q_{n-1}\right) q_{n}\right) \cong
$$




$$
\begin{aligned}
& \left.\left.\left.\left.\left.\left.\left(\ldots\left(p_{1} p_{2}\right) p_{3}\right) \ldots\right) p_{m-1}\right) p_{m}\right)\left(\ldots\left(q_{1} q_{2}\right) q_{3}\right) \ldots\right) q_{n-1}\right)\right) q_{n} \cong \\
& \left.\left.\left.\left.\left.\left.\left(\ldots\left(p_{1} p_{2}\right) p_{3}\right) \ldots\right) p_{m-1}\right) p_{m}\right)\left(\ldots\left(q_{1} q_{2}\right) q_{3}\right) \ldots\right)\right) q_{n-1}\right) q_{n} \cong \ldots \\
& \left.\left.\left.\left.\left.\left.\left.\left.\quad \cong\left(\ldots\left(p_{1} p_{2}\right) p_{3}\right) \ldots\right) p_{m-1}\right) p_{m}\right) q_{1}\right) q_{2}\right) q_{3}\right) \ldots\right) q_{n-1}\right) q_{n}
\end{aligned}
$$

Таким образом, и в этом случае произведение $p q$ имеет требуемое представление. Лемма 1 доказана.

Шаг 3. Двухполюсник $G(p)=(V(p), E(p)$, in $(p)$, out $(p))$ для терма $p$ из леммы 1 согласно определению может быть построен следующим образом.

Пусть $p \in \Lambda_{k}$, то есть $p=x_{i_{1}}\left(x_{i_{2}}\left(\ldots\left(x_{i_{k-1}} x_{i_{k}}\right) \ldots\right)\right.$. Тогда $V(p)=\left\{v_{0}, v_{1}, v_{2}, \ldots v_{k+1}\right\}, E(p)=$ $=\left\{\left(v_{i-1}, i, v_{i}\right): i=1, \ldots k\right\}$, и $\operatorname{in}(p)=v_{0}$, out $(p)=v_{k+1}$ :

$$
\operatorname{in}(p)=v_{0} \cdot \stackrel{i_{1}}{\rightarrow} \cdot \stackrel{i_{2}}{\rightarrow} \cdot \ldots \stackrel{i_{k}}{\rightarrow} \cdot v_{k} \quad \cdot v_{k+1}=\operatorname{out}(p) .
$$

Пусть $\left.\left.p=\left(\ldots\left(p_{1} p_{2}\right) p_{3}\right) \ldots\right) p_{m-1}\right) p_{m}$, где $p_{1}, p_{2}, \ldots, p_{m}$ из $\tilde{\Lambda}_{1}(m>1)$. Мы будем предполагать, что множества $V\left(p_{1}\right), V\left(p_{2}\right), \ldots, V\left(p_{m}\right)$ попарно не пересекаются. Тогда $V(p)=V\left(p_{1}\right) \cup \operatorname{pr}\left(E\left(p_{2}\right)\right) \cup$ $\cup \ldots \cup \operatorname{pr}\left(E\left(p_{m}\right)\right), E(p)=E\left(p_{1}\right) \cup E\left(p_{2}\right) \cup \ldots \cup E\left(p_{m}\right)$ и $\operatorname{in}(p)=\operatorname{in}\left(p_{1}\right), \operatorname{out}(p)=\operatorname{out}\left(p_{1}\right)$. Заметим, что в этом случае $G(p)$ содержит $m+1$ компоненту связности.

Следующая лемма непосредственно вытекает из строения соответствующих графов и определения гомоморфизма.

Лемма 2. Пусть $p=x_{i_{1}}\left(x_{i_{2}}\left(\ldots\left(x_{i_{k-1}} x_{i_{k}}\right) \ldots\right), q=x_{j_{1}}\left(x_{i_{j}}\left(\ldots\left(x_{j_{l-1}} x_{j_{l}}\right) \ldots\right) u E(p) \prec E(q)\right.\right.$. Тогда $l \leq k u j_{1}=i_{t}, j_{2}=i_{t+1}, \ldots, j_{l}=i_{t+l-1}$ для некоторого $t \in\{1, \ldots, k\}$. В частности, если $E(p) \cong E(q)$, mo $p=q$.

Лемма 3. Пусть $p \in \tilde{\Lambda}_{2}, q \in \tilde{\Lambda}_{1} u E(p) \prec E(q)$. Тогда $p \cong p q u \tilde{p} p \cong(\tilde{p} p) q$ для любого терма $\tilde{p}$.

Доказательство. Пусть $p=x_{i_{1}}\left(x_{i_{2}}\left(\ldots\left(x_{i_{k-1}} x_{i_{k}}\right) \ldots\right), q=x_{j_{1}}\left(x_{i_{j}}\left(\ldots\left(x_{j_{l-1}} x_{j_{l}}\right) \ldots\right)\right.\right.$ и $E(p) \prec E(q)$. Используя лемму 2 и тождества $(1),(2),\left(5_{k}\right)$, получаем:

$$
\begin{gathered}
p=\left(x _ { i _ { 1 } } \left(x _ { i _ { 2 } } ( \ldots ( x _ { i _ { k - 1 } } x _ { i _ { k } } ) \ldots ) \cong \left(x _ { i _ { 1 } } \left(x _ { i _ { 2 } } ( \ldots ( x _ { i _ { k - 1 } } x _ { i _ { k } } ) \ldots ) \left(x _ { i _ { 1 } } \left(x_{i_{2}}\left(\ldots\left(x_{i_{t+l-2}} x_{i_{t+l-1}}\right) \ldots\right) \cong\right.\right.\right.\right.\right.\right. \\
\cong\left(x _ { i _ { 1 } } \left(x _ { i _ { 2 } } ( \ldots ( x _ { i _ { k - 1 } } x _ { i _ { k } } ) \ldots ) \left(\left(x _ { i _ { 1 } } \left(x _ { i _ { 2 } } ( \ldots ( x _ { i _ { t + l - 2 } } x _ { i _ { t + l - 1 } } ) \ldots ) \left(x _ { i _ { 2 } } \left(x_{i_{3}}\left(\ldots\left(x_{i_{t+l-2}} x_{i_{t+l-1}}\right) \ldots\right) \cong\right.\right.\right.\right.\right.\right.\right. \\
\cong\left(\left(x _ { i _ { 1 } } \left(x _ { i _ { 2 } } ( \ldots ( x _ { i _ { k - 1 } } x _ { i _ { k } } ) \ldots ) \left(x _ { i _ { 1 } } \left(x _ { i _ { 2 } } ( \ldots ( x _ { i _ { t + l - 2 } } x _ { i _ { t + l - 1 } } ) \ldots ) \left(x _ { i _ { 2 } } \left(x_{i_{3}}\left(\ldots\left(x_{i_{t+l-2}} x_{i_{t+l-1}}\right) \ldots\right) \cong\right.\right.\right.\right.\right.\right.\right. \\
\cong\left(\left(x _ { i _ { 1 } } \left(x _ { i _ { 2 } } ( \ldots ( x _ { i _ { k - 1 } } x _ { i _ { k } } ) \ldots ) \left(x _ { i _ { 2 } } \left(x_{i_{3}}\left(\ldots\left(x_{i_{t+l-2}} x_{i_{t+l-1}}\right) \ldots\right) \cong \ldots \cong\right.\right.\right.\right.\right. \\
\cong\left(\left(x _ { i _ { 1 } } \left(x _ { i _ { 2 } } ( \ldots ( x _ { i _ { k - 1 } } x _ { i _ { k } } ) \ldots ) \left(x _ { i _ { t } } \left(x_{i_{t+1}}\left(\ldots\left(x_{i_{t+l-2}} x_{i_{t+l-1}}\right) \ldots\right)=\right.\right.\right.\right.\right. \\
=\left(\left(x _ { i _ { 1 } } \left(x _ { i _ { 2 } } ( \ldots ( x _ { i _ { k - 1 } } x _ { i _ { k } } ) \ldots ) \left(x _ { j _ { 1 } } \left(x_{i_{j}}\left(\ldots\left(x_{j_{l-1}} x_{j_{l}}\right) \ldots\right)=p q .\right.\right.\right.\right.\right.
\end{gathered}
$$

Далее, используя тождество (8), получаем $\tilde{p} p \cong \tilde{p}(p q) \cong(\tilde{p} p) q$. Лемма 3 доказана.

Лемма 4. Пусть $p \in \Lambda_{1}, q \in \tilde{\Lambda}_{1} u E(p) \prec E(q)$. Тогда $p=q u \tilde{p} p \cong(\tilde{p} p) q$ для любого терма $\tilde{p}$.

Доказательство. Так как $p \in \Lambda_{1}$, то $p=x_{i}$, следовательно, согласно лемме 2 имеем $q=p=x_{i}$. Отсюда, используя тождество (2), получаем $\tilde{p} p \cong(\tilde{p} p) p=(\tilde{p} p) q$. Лемма 4 доказана.

Шаг 4. Легко проверить, что операции * удовлетворяют тождествам (1)-(4) и $\left(5_{k}\right)$. Отсюда следует, что $\Sigma \subset E q\{*\}$. Таким образом, для доказательства теоремы достаточно показать, что всякое тождество из $E q\{*\}$ принадлежит $\Sigma$. Согласно лемме 1 мы можем предположить, что $\left.\left.p=\left(\ldots\left(p_{1} p_{2}\right) p_{3}\right) \ldots\right) p_{m-1}\right) p_{m}$ и $\left.\left.q=\left(\ldots\left(q_{1} q_{2}\right) q_{3}\right) \ldots\right) q_{n-1}\right) q_{n}$.

Предположим, что тождество $p=q$ принадлежит эквациональной теории $E q\{*\}$. Тогда согласно сформулированному выше результату из работы [5] имеем $G(p) \cong G(q)$, то есть существуют гомоморфизмы $f_{1}$ из $G(q)$ в $G(p)$ и $f_{2}$ из $G(p)$ в $G(q)$. Поскольку всякая компонента связности графа $E(q)$ при гомоморфизме $f_{1}$ будет отображаться в компоненту связности графа $E(p)$ и всякая компонента связности графа $E(p)$ при гомоморфизме $f_{2}$ будет отображаться в компоненту связности графа $E(q)$, существуют отображения $\phi$ из $\{1, \ldots, m\}$ в $\{1, \ldots, n\}$ и $\varphi$ из $\{1, \ldots, n\}$ в $\{1, \ldots, m\}$ такие, что $f\left(E\left(q_{k}\right)\right) \subset E\left(p_{\phi(k)}\right)$ для $k=1, \ldots, m$ и $f\left(E\left(p_{k}\right)\right) \subset E\left(q_{\varphi(k)}\right)$ для $k=1, \ldots, n$. Учитывая, что $f_{1}\left(i n\left(q_{1}\right)\right)=f_{1}(i n(q))=i n(p)=i n\left(p_{1}\right)$, имеем $\phi(1)=1$. Аналогично, $\varphi(1)=1$. Следовательно, $G\left(p_{1}\right) \cong G\left(q_{1}\right)$, отсюда согласно лемме 4 имеем $p_{1} \cong q_{1}$. 
Далее, используя леммы 3, 4 и тождества (6), (7), получаем:

$$
\begin{gathered}
\left.\left.\left.\left.\left.\left.p=\left(\ldots\left(p_{1} p_{2}\right) p_{3}\right) \ldots\right) p_{m-1}\right) p_{m} \cong\left(\ldots\left(p_{1}\right)^{2}\right) p_{2}\right) p_{3}\right) \ldots\right) p_{m-1}\right) p_{m} \cong \\
\left.\left.\left.\left.\left.\left.\left.\left.\cong\left(\ldots\left(p_{1} p_{1}\right) p_{2}\right) p_{3}\right) \ldots\right) p_{m-1}\right) p_{m} \cong\left(\ldots\left(p_{1} p_{1}\right) p_{2}\right) p_{3}\right) \ldots\right) p_{\phi(1)}\right) \ldots\right) p_{m-1}\right) p_{m} \cong \\
\left.\left.\left.\left.\left.\left.\left.\left.\left.\left.\cong\left(\ldots\left(p_{1} p_{1}\right) p_{2}\right) p_{3}\right) \ldots\right) p_{\phi(1)}\right) q_{1}\right) \ldots\right) p_{m-1}\right) p_{m} \cong\left(\ldots\left(p_{1} p_{1}\right) p_{2}\right) p_{3}\right) \ldots\right) p_{m-1}\right) p_{m}\right) q_{1} \cong \ldots \cong \\
\left.\left.\left.\left.\left.\left.\left.\left.\left.\left.\left.\left.\left.\cong\left(\ldots\left(p_{1} p_{1}\right) p_{2}\right) p_{3}\right) \ldots\right) p_{m-1}\right) p_{m}\right) q_{1}\right) q_{2}\right) \ldots\right) q_{n} \cong\left(\ldots\left(p_{1} p_{2}\right) p_{3}\right) \ldots\right) p_{m-1}\right) p_{m}\right) q_{1}\right) q_{2}\right) \ldots\right) q_{n} .
\end{gathered}
$$

Аналогично получаем $\left.\left.\left.\left.\left.\left.q=\left(\ldots\left(q_{1} q_{2}\right) q_{3}\right) \ldots\right) q_{n-1}\right) q_{n}\right) p_{1}\right) p_{2}\right) \ldots\right) p_{m}$. Отсюда, учитывая, что $p_{1} \cong q_{1}$, используя тождество (7), получаем $p \cong q$, то есть тождество $p=q$ принадлежит $\Sigma$. Теорема доказана.

\section{Библиограсрический список}

1. Tarski A. On the calculus of relations // J. Symbolic Logic. 1941. Vol. 4. P. 73-89.

2. Tarski A. Some methodological results concerning the calculus of relations // J. Symbolic Logic. 1953. Vol. 18. P. 188-189.

3. Бредихин Д. А. Об алгебрах отношений с диофантовыми операциями // Докл. АН. 1998. Т. 360. С. 594595.

4. Böner P., Pöschel F. R. Clones of operations on binary relations // Contributions to general algebras. 1991. Vol. 7. P. 50-70.

5. Бредихин Д. А. Эквациональная теория алгебр отношений с позитивными операциями // Изв. вузов. Математика. 1993. № 3. С. 23-30.

6. Andreka H., Bredikhin D. A. The equational theory of union-free algebras of relations // Alg. Univers. 1994. Vol. 33. P. 516-532.

7. Бредихин Д. А. О квазитождествах алгебр отношений с диофантовыми операциями // Сиб. мат. журн. 1997. T. 38. C. 29-41.

8. Bredikhin D. A. On relation algebras with general superpositions // Colloq. Math. Soc. J. Bolyai. 1994. Vol. 54. P. 11-124.

9. Bredikhin D. A. Varietes of groupoids associated with involuted restrictive bisemigroups of binary relations // Semigroup Forum. 1992. Vol. 44. Р. 87-192.

10. Бредихин Д. А. О многообразии группоидов бинарных отношений // Изв. Сарат. ун-та. Нов. сер. Сер. Математика. Механика. Информатика. 2013. Т. 13, вып. 1, ч. 1. С. $93-98$.

11. Henkin L., Monk J. D., Tarski A. Cylindric Algebras. North-Holland, Amsterdam, 1971. 311 p.

\section{On Classes of Groupoids of Relations with Diophantine Operations}

\section{A. Bredikhin}

Saratov State Technical University, Russia, 410054, Politechnicheskaya st., 77, bredikhin@mail.ru

In the paper the bases of identities of varieties generated by classes of groupoids of the binary relations with diophantine operations are found.

Key words: algebra of relations, diophantine operations, identities, varieties, groupoids.

\section{References}

1. Tarski A. On the calculus of relations. J. Symbolic Logic, 1941, vol. 4, pp. 73-89.

2. Tarski A. Some methodological results concerning the calculus of relations. J. Symbolic Logic, 1953, vol. 18. pp. $188-189$.

3. Bredikhin D. A. On algebras of relations with Diophantine operations. Doklady Mathematics, 1998, vol. 57, no. 3, pp. 435-436.

4. Böner P., Pöschel F. R. Clones of operations on binary relations. Contributions to general algebras, 1991, vol. 7, pp. 50-70.

5. Bredikhin D. A. The equational theory of algebras of relations with positive operations. Rus. Math. (Izvestiya VUZ. Matematika), 1993, vol. 37, no. 3, pp. 21-28.

6. Andreka H., Bredikhin D. A. The equational theory of union-free algebras of relations. Alg. Univers., 1994, vol. 33, pp. 516-532.

7. Bredikhin D. A. On quasi-identities of algebras of relations with diophantine operations. Siberian Mathematical Journal, 1997, vol. 38, no. 1, pp. 23-33

8. Bredikhin D. A. On relation algebras with general superpositions. Colloq. Math. Soc. J. Bolyai, 1994, vol. 54, pp. 11-124. 
9. Bredikhin D. A. Varietes of groupoids associated with involuted restrictive bisemigroups of binary relations. Semigroup Forum, 1992, vol. 44. pp. 87-192.

10. Bredikhin D. A. On varieties of groupoids of binary relations. Izv. Sarat. Univ. N.S. Ser. Math. Mech. Inform., 2013, vol. 13, iss. 1, pt. 1, pp. 13-21 (in Russian). 11. Henkin L., Monk J. D., Tarski A. Cylindric Algebras. North-Holland, Amsterdam, 1971, 311 p.

УДК 511

\title{
ОБ АРИФМЕТИЧЕСКИХ СВОЙСТВАХ ОБОБЩЕННОЙ ПОСЛЕДОВАТЕЛЬНОСТИ ФИБОНАЧЧИ И ИХ СЛЕДСТВИЯХ
}

\begin{abstract}
А. Н. Васильев
Преподаватель кафредры математики и инсрорматики, Казахстанский срилиал Московского государственного университета им. М.В.Ломоносова, г. Астана, Республика Казахстан, antonvassilyev@ mail.ru

В работе изучены некоторые свойства распределения членов обобщенной последовательности Фибоначчи по бесквадратному модулю и получены следствия из этих свойств.
\end{abstract}

Ключевые слова: обобщенная последовательность Фибоначчи, тригонометрические суммы, плотность множества.

\section{1. СВОЙСТВА ОБОБЩЕННОЙ ПОСЛЕДОВАТЕЛЬНОСТИ ФИБОНАЧЧИ}

Последовательность Фибоначчи, как известно, задается следующим образом: $F_{1}=1, F_{2}=1$, $F_{n+2}=F_{n+1}+F_{n}$. Обобщенная последовательность Фибоначчи задается тем же рекуррентным соотношением и двумя начальными натуральными членами, т. е. $G_{1}=a, G_{2}=b, G_{n+2}=G_{n+1}+G_{n}$, где $a, b$ - натуральные числа. Вторую последовательность на протяжении всей работы будем считать наперед заданной.

Пусть, на протяжении всей работы, $d$ - бесквадратное (не делящееся ни на какой квадрат простого) натуральное число, большее 1 и взаимно простое с числами $a, b$ и с числом $\left(a^{2}+a b-b^{2}\right)$ (это экзотическое условие будет мотивировано позже). Через $p$ будем обозначать, как обычно, простое число. В первой части это будут простые, взаимно простые с числами $a, b$ и с числом $\left(a^{2}+a b-b^{2}\right)$. Во второй части простые, выступающие делителями какого-нибудь $d$, также будут предполагаться удовлетворяющими этому дополнительному условию.

Введем малый $d$-период последовательности Фибоначчи $t(d)=\min \left\{\tau: \tau \geq 1, d \mid F_{\tau}\right\}$ и большой $d$-период последовательности Фибоначчи $T(d)=\min \left\{\mathrm{T}: \mathrm{T} \geq 1, F_{n+\mathrm{T}} \equiv F_{n}(\bmod d) \forall n\right\}$. Аналогично, большой $d$-период обобщенной последовательности Фибоначчи есть $T^{\prime}(d)=\min \{\mathrm{T}: \mathrm{T} \geq 1$, $\left.G_{n+\mathrm{T}} \equiv G_{n}(\bmod d) \forall n\right\}$ (периодичность по любому модулю доказывается просто). Аналога малого $d$-периода может не существовать (например, если $a=2, b=1, d=5$ ).

Выделим необходимые нам свойства последовательности Фибоначчи в следующую лемму.

Лемма 1.1.

A) $F_{n}=\frac{(2)(2)}{\sqrt{5}}$ (формула Бине).

Б) $F_{n+m}=F_{n-1} F_{m}+F_{n} F_{m+1}$.

B1) $d\left|F_{n} \Leftrightarrow t(d)\right| n$.

B2) $\left\{\begin{array}{l}F_{\alpha} \equiv F_{\beta}(\bmod d), \\ F_{\alpha+1} \equiv F_{\beta+1}(\bmod d)\end{array} \quad \Leftrightarrow T(d) \mid(\alpha-\beta)\right.$.

Г) $T(d) / t(d) \in\{1,2,4\}$.

Д) $d=p_{1} p_{2} \ldots p_{s} \Rightarrow t(d)=\left[t\left(p_{1}\right), t\left(p_{2}\right), \ldots, t\left(p_{s}\right)\right]$.

(C) Васпльев А. Н., 2013 